\title{
Kognitiv, kognitiv-verhaltens- therapeutisch, metakognitiv: Wer blickt da noch durch? Ein Update zu kognitiven Psychotherapieansätzen
}

\author{
Lena Jelinek und Steffen Moritz
}

Universitätsklinikum Hamburg-Eppendorf, Zentrum für Psychosoziale Medizin, Klinik und Poliklinik für Psychiatrie und Psychotherapie

\begin{abstract}
Zusammenfassung: Allen kognitiv-verhaltenstherapeutischen Ansätzen, ob von der sogenannten zweiten oder dritten Welle, ist die Arbeit an den Kognitionen gemein. Damit nimmt die kognitive Arbeit eine zentrale Funktion in den verschiedenen Verfahren ein. In unserer Einführung werden die im Themenheft näher ausgeführten Ansätze und ihre Gemeinsamkeiten dargestellt.
\end{abstract}

Schlüsselwörter: kognitive Verhaltenstherapie, KVT, Dritte Welle, Psychotherapie, Kognition

Cognitive, cognitive-behavioral, metacognitive: How to stay on top of things? An update on cognitive interventions

Abstract: To work on the client's cognitions is central to cognitive-behavioral therapy (CBT). No matter what wave (or generation) of approaches we are looking at. In this introduction to the current special issue, the different approaches presented in the issue are briefly outlined and common features are highlighted.

Keywords: Cognitive-behavioral therapy, CBT, third wave, psychotherapy, cognition

Die Verhaltenstherapie wird seit den 1950er Jahren zur Behandlung psychischer Störungen eingesetzt und hat sich seitdem beständig weiterentwickelt. Im Fokus stand zunächst die Anwendung von Lernprinzipien auf beobachtbares Problemverhalten. Später wurde die Verhaltenstherapie um kognitive Konzepte erweitert, beispielsweise bezüglich der Annahmen über die Entstehung sowie die Behandlung psychischer Störungen. Entsprechend wurde sie fortan als Kognitive Verhaltenstherapie (KVT) bezeichnet. Der nächste Schritt der Weiterentwicklung wird auch als „dritte Welle“ der Verhaltenstherapie bezeichnet (Hayes, 2004) und reicherte das Repertoire der kognitiven Verhaltenstherapie um Themen wie Achtsamkeit, Emotionen, Akzeptanz und Werte an (Hayes \& Hofmann, 2017). Damit hielten nicht nur neue Methoden und therapeutische Strategien Einzug in die Verhaltenstherapie. Es wurden auch umfassende an die KVT angelehnte Therapiekonzepte, wie beispielsweise die Mindfulness Based Cognitive Therapy (MBCT, dt. Achtsamkeitsbasierte Kognitive Therapie), die Acceptance and Commitment Therapy (ACT) oder das Cognitive Behavioral Analysis System of Psychotherapy (CBASP) entwickelt (Heidenreich \& Michalak, 2013). Darüber hinaus führte die stetige Weiterentwicklung der kognitiven Verhaltenstherapie zur Ausweitung der Indikation dieses Richtlinienverfahrens (z.B. für Psychose).

Wie bereits dargestellt, begann die kognitive Orientierung mit der zweiten „Generation“ der Verhaltenstherapie und die Leserinnen und Leser fragen sich vielleicht, warum wir den Fokus des aktuellen Themenhefts auf das Kognitive gelegt haben, obwohl die in dem Heft beschriebenen Therapieansätze überwiegend der dritten Welle der KVT zugeschrieben werden und viele der Schulen den Fokus explizit auf andere Aspekte legen (z.B. Emotionen, Werte). Als Herausgebende war uns wichtig, die kognitiven Aspekte dieser Ansätze zu charakterisieren und damit herauszustellen, dass trotz aller Weiterentwicklungen und der Ergänzung neuer Aspekte, die Arbeit an den Kognitionen eine wichtige Schnittstelle der verschiedenen Ansätze darstellt. Dabei geht die kognitive Arbeit sicher oft über das hinaus, was in der kognitiven Wende darunter verstanden wurde (u.a. die Disputation von Überzeugungen, sokratischer Dialog) und bezieht metakognitive Aspekte und Prozesse sowie Emotionen mit ein oder fokussiert auf die Behandlung kognitiver Symptome, wie z.B. das Grübeln. Dennoch bleibt die 
kognitive Arbeit eine der zentralen Gemeinsamkeiten dieser Verfahren, deren Verfechter bedauerlicherweise gelegentlich in Konkurrenz zueinander stehen. Mit dem aktuellen Themenheft hoffen wir, Ihnen diese Gemeinsamkeiten zu vermitteln und Ihnen ein Update zum Stand der nachfolgend dargestellten Therapieansätze zu geben.

\section{Inhalte des Themenschwerpunktes}

Das Themenheft wird eröffnet mit dem Überblicksartikel zur Ruminations-Fokussierten Kognitiven Verhaltenstherapie (engl. Rumination-Focused Cognitive Behavioral Therapy, RFCBT) von Ehring (2020). Anhand eines Fallbeispiels stellt er einen therapeutischen Ansatz vor, der sich vorrangig der Behandlung von Grübeln als einer mentalen (oder auch kognitiven) „Gewohnheit“ widmet, welche unter anderem der Vermeidung dient. Hier werden etablierte Techniken wie die Verhaltensanalyse und die Selbstbeobachtung mit neuen Strategien kombiniert (z.B. Training im konkreten Denken). Die bisherigen Wirksamkeitsnachweise von der RFCBT beziehen sich vorrangig auf Patientinnen und Patienten mit einer Depression. Ehring (2020) selbst stellt die Frage, ob, aufgrund der fehlenden traditionellen KVT-Elemente (z.B. inhaltliche Disputation), die RFCBT eine "kognitive“ Therapie darstellt und betont die Gemeinsamkeiten zu anderen Verfahren der dritten Welle. Hierbei bezieht Ehring (2020) sich beispielsweise auf Verfahren, die auf die Behandlung von Metakognitionen fokussieren.

Was unter Metakognition zu verstehen ist und welche metakognitiven Elemente sich auch in der traditionellen KVT finden lassen, wird im Beitrag von Dietrichkeit et al. (2020) ausführlich erläutert. Es werden unterschiedliche Interventionen vorgestellt, welche explizit den Begriff ,Metakognition" im Namen tragen. Im Detail stellen Dietrichkeit et al. (2020) das Metakognitive Training (MKT) am Beispiel des Metakognitiven Trainings bei Depression (D-MKT) dar. Hierbei handelt es sich um ein standardisiertes Gruppenkonzept, welches in acht präsentationsgestützten Modulen unter Verwendung von spielerischen Übungen den Patientinnen und Patienten depressionstypische kognitive Verzerrungen sowie deren Veränderbarkeit vermittelt. Der Artikel schließt mit bisher vorliegenden Wirksamkeitsnachweisen sowie der Darstellung von zwei spezifischen Weiterentwicklungen für ältere Menschen und Geflüchtete.

Die Metakognitive Therapie (engl. Metacognitive Therapy, MCT) wird von Hansmeier und Exner (2020) vorgestellt. Zunächst werden die theoretischen Grundlagen sowie deren Anpassung für unterschiedliche Störungsbilder beschrieben. Anschließend werden konkrete Techniken präsentiert (wie z.B. das Aufmerksamkeitstraining und die Losgelöste Achtsamkeit), bevor die bisher vorliegenden Wirksamkeitsnachweise von der MCT dargelegt werden. Die Befunde weisen darauf hin, dass die MCT eine wirksame und aufgrund der geringen Sitzungszahl vor allem ökonomische Behandlungsoption darstellt.

Die in der Überblicksarbeit von Zens und Jacob (2020) dargestellte Schematherapie wurde bereits in den 1990er Jahren von Jeffrey Young auf der Basis der KVT entwickelt und repräsentiert damit das älteste der in diesem Sonderheft vorgestellten Therapiekonzepte. Sie mag aufgrund ihrer „Teilearbeit" und den dazugehörigen erlebnisorientierten Techniken (z.B. Stuhldialoge) auf den ersten Blick nicht wie eine kognitive Intervention wirken. Bei genauerer Betrachtung sind die kognitiven Anteile jedoch gut erkennbar, sowohl in der Konzeption der Störungsentstehung als auch in der von Zens und Jacob (2020) dargestellten Vielzahl von traditionellen KVT Methoden, die eingesetzt werden, um Schemata zu modifizieren (z. B. Pro- und Kontra-Listen).

Das Themenheft schließt mit zwei Studien von Klein et al. (2020), die sich dem präoperatorischen Denkstil widmen, der nach dem Modell des Cognitive Behavioral Analysis System of Psychotherapy (CBASP) vor allem bei Menschen mit einer chronischer Depression zu beobachten ist und der den Zusammenhang zwischen widrigen Kindheitserfahrungen und feindseligen Verhaltensweisen mediiert. Klein et al. (2020) untersuchten den präoperativen Denkstil bei unterschiedlichen (klinischen) Stichproben (Depression, Angst- und Schmerzstörungen) im Vergleich $\mathrm{zu}$ gesunden Kontrollen und leiten theoretische sowie klinische Implikationen in Bezug auf das CBASP ab.

Bei all den geschilderten Weiterentwicklungen wurde in bester verhaltenstherapeutischer Tradition nicht nur Wert auf ein evidenzbasiertes theoretisches Fundament gelegt, sondern auch auf eine Überprüfung der Wirksamkeit und Wirkweise der Konzepte. Vermutlich ist darin das treibende Motiv für die stetigen Weiterentwicklungen zu suchen, denn trotz der Effektivität der (kognitiven) Verhaltenstherapie führen die vorliegenden Behandlungskonzepte nur selten zu einer Vollremission bzw. nicht alle Personen sprechen auf die jeweilige Therapie an. Aktuelle Bemühungen hinsichtlich der Optimierung beschäftigen sich vor allem damit, die Veränderungsmechanismen sowie die Wirksamkeit der einzelnen Bestandteile genauer zu beleuchten. Ein weiterer Fokus liegt aber auch darauf, wie die Behandlungen personalisiert werden können und unter welchen Bedingungen welche Intervention für welche Person optimal ist. Ideen zur Erweiterung der Forschungsmethodik sind in den letzten Jahren vor allem aus der Perspektive der Prozessbasierten Kognitiven Verhaltenstherapie formuliert worden (Hayes \& Hofmann, 2018; Hayes et al., 2019). 
Wir hoffen mit diesem Themenheft mit dem Schwerpunkt auf kognitive Therapien, einen aktuellen Überblick über den Stand in Theorie, Praxis und Evidenz sowie auf zukünftige Perspektiven in diesem Feld zu geben.

\section{Literatur}

Dietrichkeit, M., Moritz, S. \& Jelinek, L. (2020). Die Behandlung psychischer Störungen mittels metakognitiver Interventionen am Beispiel des Metakognitiven Trainings für Depression (D-MKT). Zeitschrift für Psychiatrie, Psychologie und Psychotherapie, 68, $160-170$

Ehring, T. (2020). Die Ruminationsfokussierte Kognitive Verhaltenstherapie. Zeitschrift für Psychiatrie, Psychologie und Psychotherapie, 68, 150-159.

Hansmeier, J. \& Exner, C. (2020). Störungs- und Behandlungskonzepte sowie empirische Evidenz der metakognitiven Therapie nach Wells. Zeitschrift für Psychiatrie, Psychologie und Psychotherapie, 68, 171-179.

Hayes, S. C. (2004). Acceptance and commitment therapy, relational frame theory, and the third wave of behavioral and cognitive therapies. Behavior Therapy, 47, 869-885.

Hayes, S. C. \& Hofmann, S. G. (2017). The third wave of cognitive behavioral therapy and the rise of process-based care. World Psychiatry, 16, 245-246.
Hayes, S. C. \& Hofmann, S. G. (2018). Process-based CBT: The science and core clinical competencies of cognitive behavioral therapy. Oakland, CA: New Harbinger Publications.

Hayes, S. C., Hofmann, S. G., Stanton, C. E., Carpenter, J. K., Sanford, B. T., Curtiss, J. E. et al. (2019). The role of the individual in the coming era of process-based therapy. Behaviour Research and Therapy, 117, 40-53.

Heidenreich, T. \& Michalak, J. (2013). Die »dritte Welle« der Verhaltenstherapie: Grundlagen und Praxis. Weinheim: Beltz.

Klein, J. P., Grasshoff, L., Hermanns, R., Haeger, S., Sondermann, S. Kühnen, T. et al. (2020). Präoperatorisches Denken bei Patient_innen mit Angststörung oder Chronischem Rückenschmerz: Zwei Querschnittsstudien zum besseren Verständnis des Cognitive Behavioral Analysis System of Psychotherapy (CBASP). Zeitschrift für Psychiatrie, Psychologie und Psychotherapie, 68, 191-199.

Zens, C. \& Jacob, G. (2020). Grundlagen und therapeutisches Vorgehen in der Schematherapie. Zeitschrift für Psychiatrie, Psychologie und Psychotherapie, 68, 180-190.

\section{Prof. Dr. Lena Jelinek}

Universitätsklinikum Hamburg-Eppendorf, Zentrum für Psychosoziale Medizin

Klinik und Poliklinik für Psychiatrie und Psychotherapie Martinistraße 52

20246 Hamburg

Deutschland

ljelinek@uke.de 